\title{
Sewage effluent from an Indian hospital harbors novel carbapenemases and integron-borne antibiotic resistance genes
}

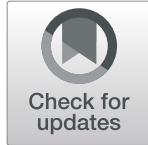

\author{
Nachiket P. Marathe ${ }^{1,2,3}$, Fanny Berglund ${ }^{1,4}$, Mohammad Razavi ${ }^{1,2}$, Chandan Pal $^{1,5}$, Johannes Dröge ${ }^{4}$, \\ Sharvari Samant ${ }^{6}$, Erik Kristiansson ${ }^{1,4}$ and D. G. Joakim Larsson ${ }^{1,2^{*}}$
}

\begin{abstract}
Background: Hospital wastewaters contain fecal material from a large number of individuals, of which many are undergoing antibiotic therapy. It is, thus, plausible that hospital wastewaters could provide opportunities to find novel carbapenemases and other resistance genes not yet described in clinical strains. Our aim was therefore to investigate the microbiota and antibiotic resistome of hospital effluent collected from the city of Mumbai, India, with a special focus on identifying novel carbapenemases.
\end{abstract}

Results: Shotgun metagenomics revealed a total of 112 different mobile antibiotic resistance gene types, conferring resistance against almost all classes of antibiotics. Beta-lactamase genes, including encoding clinically important carbapenemases, such as NDM, VIM, IMP, KPC, and OXA-48, were abundant. NDM (0.9\% relative abundance to $16 \mathrm{~S}$ rRNA genes) was the most common carbapenemase gene, followed by OXA-58 (0.84\% relative abundance to $16 \mathrm{~S}$ rRNA genes). Among the investigated mobile genetic elements, class 1 integrons (11\% relative abundance to 165 rRNA genes) were the most abundant. The genus Acinetobacter accounted for as many as 30\% of the total $16 \mathrm{~S}$ rRNA reads, with A. baumannii accounting for an estimated $2.5 \%$. High throughput sequencing of amplified integron gene cassettes identified a novel functional variant of an IMP-type (proposed IMP-81) carbapenemase gene (eight aa substitutions) along with recently described novel resistance genes like sul4 and bla $a_{\text {RSA }}$. Using a computational hidden Markov model, we detected 27 unique metallo-beta-lactamase (MBL) genes in the shotgun data, of which nine were novel subclass B1 genes, one novel subclass B2, and 10 novel subclass B3 genes. Six of the seven novel MBL genes were functional when expressed in Escherichia coli.

Conclusion: By exploring hospital wastewater from India, our understanding of the diversity of carbapenemases has been extended. The study also demonstrates that the microbiota of hospital wastewater can serve as a reservoir of novel resistance genes, including previously uncharacterized carbapenemases with the potential to spread further.

Keywords: Antibiotic resistance, Hospital effluent, Carbapenemase, Pathogens, Hidden Markov model, India

\section{Background}

The rise in antibiotic resistance is a serious growing problem for human health [1]. Environmental and commensal microbiota serves as sources for antibiotic resistance genes (ARGs) that emerge over time in pathogens

\footnotetext{
* Correspondence: joakim.larsson@fysiologi.gu.se

'Centre for Antibiotic Resistance Research (CARe), University of Gothenburg, Gothenburg, Sweden

${ }^{2}$ Department of Infectious Diseases, Institute of Biomedicine, Sahlgrenska Academy, University of Gothenburg, Gothenburg, Sweden

Full list of author information is available at the end of the article
}

through horizontal gene transfer [2, 3]. Characterization of the environmental resistome, thus, would provide an understanding of novel resistance factors that might be encountered in clinics in the future. This in turn would help to better understand the development of resistance in pathogens and to prepare surveillance and control measures to reduce their dissemination.

Recently, hidden Markov model (HMM)-based methods have been developed for detecting ARGs from shotgun sequence data [4-7]. We have successfully

(c) The Author(s). 2019 Open Access This article is distributed under the terms of the Creative Commons Attribution 4.0 International License (http://creativecommons.org/licenses/by/4.0/), which permits unrestricted use, distribution, and 
applied HMM to identify novel quinolone resistance genes $[8,9]$ as well as 59 novel families of the subclass B1 metallo-beta-lactamases (MBLs) capable of degrading carbapenems from a variety of environments [10]. HMM accurately predicts the gene fragments belonging to specific gene classes, based on evolutionarily conserved domains [11]. This leads to accurate detection of both known and previously undescribed resistance genes in genomic and metagenomic sequence data. Functional metagenomics is another strategy that has the ability to identify novel ARGs without apparent similarities to known ARGs as it is based on a functional selection of DNA fragments expressed in a surrogate host like Escherichia coli [12]. With such an approach, we have identified novel ARGs, including one encoding a carbapenem hydrolyzing beta-lactamase, from river sediments contaminated with drug production waste [13]. Several studies, using functional metagenomics, have reported novel resistance genes from a variety of environments like human gut, soil, and seawater [14-18].

Neither approach relies on the host-bacteria being cultivable, but both largely lack the ability to differentiate between mobilized and non-mobilized genes. The latter is a critical aspect for assessing risk associated with ARGs [19, 20]. To overcome this, we have recently used a high-throughput sequencing method for studying genes associated with integrons [21]. Class 1 integrons are frequently carried by human pathogens and very often harbor ARGs. The integrons are usually located on mobile genetic elements like plasmids and transposons, providing the ability to move across cells, strains, and species [22-24]. Using an approach of amplifying partial class 1 integrons, the fourth mobile sulfonamide resistance gene (sul4) was discovered together with several other novel resistance genes, including those encoding class $\mathrm{D}$ beta-lactamases conferring reduced susceptibility to carbapenems in E. coli [21].

Hospital sewage represents a collection of fecal matter of a large number of individuals including patients undergoing antibiotic treatment, together with other bacteria of environmental origin. Antibiotic residues in hospital wastewaters may reach levels that potentially could be selective for resistant strains [25-27]. Several studies have accordingly shown that hospital effluents can provide a rich variety of known ARGs and resistant enteric pathogens [28-32]. It is therefore plausible that a more explorative analysis could lead to the discovery of novel ARGs, either easily accessible to pathogens or already circulating among pathogens undetected.

Antibiotic resistance is a growing problem in India, partly due to high consumption of broad-spectrum antibiotics, including carbapenems [1]. Both clinical and environmental resistance has been on the rise in India in the last decade [33]. For example, the incidences of carbapenem resistance in Klebsiella isolates and the rate of methicillin-resistant Staphylococcus aureus (MRSA) infections have gone up in the last few years [20]. Nosocomial infections with carbapenemase-producing organisms (CPOs) are quite common in India [34], and studies have shown that hospital environments are a common reservoir of CPOs [28, 29]. A recent Indian study has shown that hospital effluent contributes to the spread of carbapenemases in the external environment [30]. For these reasons, it is possible that the Indian hospital effluent could provide a particularly rich variety of carbapenemases, including not yet characterized forms.

With this background, we aimed at identifying and characterizing novel ARGs in wastewater from an Indian hospital, particularly with regards to carbapenemases. This was achieved using two approaches-(1) investigating the antibiotic resistome and microbiota of sewage effluent collected from a large hospital in the city of Mumbai in India using Illumina HiSeq-based shotgun metagenomics and identification of novel molecular class B carbapenemases from the shotgun data using hidden Markov models, and (2) investigating novel mobile resistance genes by targeted amplicon sequencing of integron gene cassettes using a combination of shortread (Illumina MiSeq) and long-read (PacBio) sequencing technologies followed by homology searches to known ARGs. With these approaches, we detected several recently discovered resistance genes as well as genes encoding previously uncharacterized carbapenemases which we also functionally verified in $E$. coli.

\section{Results}

Shotgun sequencing of the hospital effluent metagenome resulted in $1.72 \times 10^{8}$ filtered reads. A total of 193,098 reads $(0.11 \%$ of the total reads) matched to ARGs. Together, these accounted for 0.844 copies of ARGs per $16 \mathrm{~S}$ rRNA gene. One hundred and twelve different mobile ARG types conferring resistance against almost all major classes of antibiotics were detected (Additional file 1: Table S1).

\section{Carbapenemases, class 1 integrase, and virulence genes were common in hospital effluent}

The sulfonamide resistance gene sul1 (11.4\% relative abundance to $16 \mathrm{~S}$ rRNA genes) was the most abundant resistance gene followed by the macrolide resistance gene mphE (11.3\% relative abundance to $16 \mathrm{~S}$ rRNA genes). Beta-lactamases were the most abundant gene class detected followed by aminoglycoside and tetracycline resistance genes (Additional file 1: Table S1). Twenty-seven different beta-lactamase genes (Fig. 1, Additional file 1: Table S1) were detected in the 


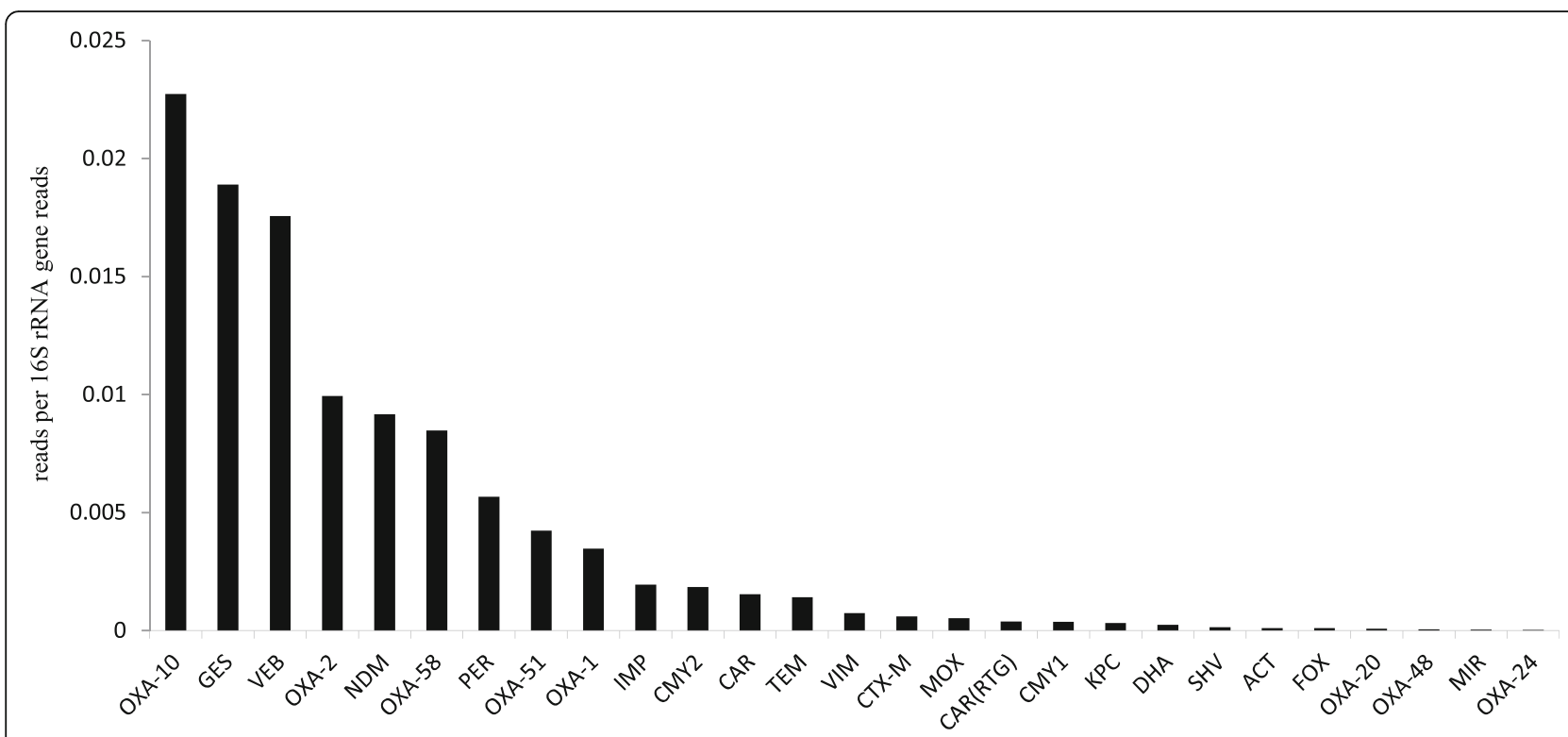

Fig. 1 Relative abundance of different beta-lactamase genes detected in hospital effluent

metagenome followed by 19 different genes conferring resistance against aminoglycosides and tetracyclines. OXA-10 and GES-type ESBLs were the dominant betalactamases detected in the study. The detected betalactamase genes also included those encoding clinically important carbapenemases, such as NDM, VIM, IMP, $\mathrm{KPC}$, and OXA-48. Among the carbapenemases, NDM had the highest abundance $(0.9 \%$ relative abundance to 16S rRNA genes), which corresponds to approximately 1 in 25 bacterial cells carrying NDM, if we assume on an average of 4 copies of the $16 \mathrm{~S}$ rRNA gene per genome in the microbial community $[35,36]$. OXA-58 $(0.84 \%$ relative abundance to $16 \mathrm{~S}$ rRNA genes) was the second most abundant carbapenemase, while OXA-48 and OXA-24 had the lowest abundance $(0.005 \%$ and $0.0025 \%$ relative abundance to $16 \mathrm{~S}$ rRNA genes, respectively). Among the studied mobile genetic elements, class 1 integrase $(10.9 \%$ relative abundance to $16 \mathrm{~S}$ rRNA genes) was the most common gene followed by ISCR2 and ISCR5 (Additional file 1: Table S1). Virulence genes involved in pilus formation, capsule formation, proteases, siderophore production, adhesion factors, and toxins like cytolysin and hemolysin were also detected (Additional file 2: Table S2). Some virulence genes specific to certain pathogens including enteropathogenic E. coli (protease stcE, intimin), Salmonella typhi (Vi antigen synthesis genes tviBC), Staphylococcus aureus (clumping factor A clfA), Streptococcus pneumoniae (fibronectin-binding protein $f b p 54$ ), and several virulence genes for Pseudomonas aeruginosa were detected [37-40], indicating the presence of these pathogens in the effluent sample.

\section{Acinetobacter baumannii was abundant in hospital effluent}

At the phylum level, Proteobacteria dominated (75\% of all 16S rRNA gene reads) followed by Bacteroidetes (16\%) and Firmicutes (1.75\%). Within Proteobacteria, Gammaproteobacteria were the most abundant, followed by Betaproteobacteria. At genus level, Acinetobacter was found to be the most dominant genera representing $30 \%$ of the $16 \mathrm{~S}$ rRNA reads (Additional file 3: Table S3). The abundance of the OXA-51 gene, which is a characteristic for $A$. baumannii [41], was $0.4 \%$ in relation to the total number of $16 \mathrm{~S}$ rRNA reads. Acinetobacter contains 6 copies of $16 \mathrm{~S}$ rRNA gene per genome [42]. This suggests that around $8 \%$ of total Acinetobacter (2.4\% of the all $16 \mathrm{~S}$ reads) detected in the samples belong to A. baumannii, thus, potentially representing pathogenic Acinetobacter strains. Further, the presence of $A$. baumannii was confirmed by alignment of the OXA-51 gene containing contigs from the assembled shotgun sequence data to $A$. baumannii genome that resulted in a perfect match. The referencebased assembly using A. baumannii genome sequences contained 1704 contigs (min 1 kilobases) with a total of 4.9 megabases. A single 9203 base pair long contig with 28-fold coverage matched to the original OXA-51 subsequence and was then used to identify the single closest known full genome of $A$. baumannii strain AB6200 (accession NZ_CP010397.1, 99\% nucleotide identity). An alignment of the assembled contigs against this genome sequence using D-Genies (http://dgenies.toulouse.inra.fr/) [43] showed an extensive coverage of the genome by assembled contigs with high matching identity (Additional file 9: Figure S1). 
Carbapenemases and novel resistance genes, including a novel IMP variant, were detected in integron gene cassettes

Sequencing of gene cassette amplicons resulted in 106, 851 long PacBio reads with an average length of 1.5 kilobases and 14,184,598 short Illumina reads (SRs) with a maximum length of 250 bases. A total of 250,720 open reading frames (ORFs) were identified. Clustering of all identified ORFs at 99\% amino acid identity led to 6098 unique ORFs. The list of genes detected in class 1 integron gene cassettes can be found in Additional file 4: Table S4, and the list of known and putative novel ARGs detected in class 1 integron gene cassettes can be found in Additional file 5: Table S5. Several carbapenemases including DIM-1, IMP-1, IMP-15, IMP-6, VIM-2, and GES-type beta-lactamases were detected, along with several putative novel resistance genes/gene variants (Table 1). We also detected many variants of GES (GES2, GES-4, GES-5, GES-6, GES-14) capable of carbapenem hydrolysis in gene cassettes from hospital effluent. Recently reported ARGs like bla $a_{\mathrm{RSA} 1}$ and sul4 were also found as part of the gene cassettes. Interestingly, we detected a novel variant of an IMP-type carbapenemase. This novel variant (proposed-IMP-81) has 8 aa substitutions compared to the closest known variant of IMP-15 (Fig. 2). This gene provided reduced susceptibility against carbapenems when expressed in $E$. coli (Additional file 6: Table S6) and was positive for imipenem degradation in the CarbaNP test.

\section{Novel class B carbapenemase genes outnumbered previously characterized carbapenemases in the hospital effluent microbiota}

We applied a recently developed computational method based on hidden Markov models to predict novel subclass B1, B2, and B3 carbapenemases directly from the shotgun data. In total, we detected 14 unique ORFs representing subclass $B 1$, one unique ORFs representing B2, and 12 unique ORFs representing subclass B3, respectively (Table 2). The phylogenetic trees for the subclass B1/B2 and subclass B3 genes detected are presented as Additional file 10: Figure S2 and Additional file 11: Figure S3. Out of the 27 unique ORFs, seven represented previously characterized genes, viz NDM-1,

Table 1 Putative novel resistance genes and gene variants detected in integron gene cassettes

\begin{tabular}{|c|c|c|c|c|c|}
\hline Gene name & $\begin{array}{l}\text { Length } \\
\text { (amino acid) }\end{array}$ & Closest homologue in NCBI protein database & $\begin{array}{l}\text { Identity } \\
(\%)\end{array}$ & $\begin{array}{l}\text { Accession } \\
\text { number }\end{array}$ & $\begin{array}{l}\text { Resistance } \\
\text { against }\end{array}$ \\
\hline aadA- like-1 & 288 & Aminoglycoside adenyltransferase, partial [Escherichia coli] & 89 & AEG64741.1 & Aminoglycosides \\
\hline sat-like-1 & 301 & $\begin{array}{l}\text { Streptomycin 3-adenylyltransferase [Yersinia pestis biovar } \\
\text { Orientalis str. IP275] }\end{array}$ & 94 & EDR32758.1 & Aminoglycosides \\
\hline aadA- like-2 & 287 & Aminoglycoside adenyltransferase, partial (plasmid) [Escherichia coli] & 94 & AlL81899.1 & Aminoglycosides \\
\hline CAT-like & 170 & Chlor_Acetyltrans_CAT, partial [uncultured bacterium] & 92 & AMP56890.1 & Amphenicols \\
\hline$b / a_{\mathrm{RSA}}{ }^{*}$ & 270 & $\begin{array}{l}\text { Class A extended-spectrum beta-lactamase BEL-1 } \\
\text { [Pseudomonas aeruginosa] }\end{array}$ & 53 & WP_063857830.1 & Beta-lactams \\
\hline OXA-like-1 & 300 & Class D [uncultured bacterium] & 87 & AMP47162.1 & Beta-lactams \\
\hline OXA-like-2 & 266 & Class D [uncultured bacterium] & 91 & AMP48561.1 & Beta-lactams \\
\hline OXA-like-3 & 266 & $\begin{array}{l}\text { Oxacillin-hydrolyzing class D beta-lactamase OXA-10 } \\
\text { [Gammaproteobacteria] }\end{array}$ & 92 & WP_000846390.1 & Beta-lactams \\
\hline OXA-like-4 & 266 & $\begin{array}{l}\text { Oxacillin-hydrolyzing class D beta-lactamase OXA-119 } \\
\text { [Pseudomonas aeruginosa] }\end{array}$ & 93 & WP_032490445.1 & Beta-lactams \\
\hline $\begin{array}{l}\text { AmpC-like- } \\
1\end{array}$ & 329 & Beta-lactamase [Vibrio cholerae non-01/non-0139] & 94 & BAE71359.1 & Beta-lactams \\
\hline OXA-like-5 & 275 & Oxacillin-hydrolyzing class D beta-lactamase OXA-2 [Proteobacteria] & 95 & WP_001007673.1 & Beta-lactams \\
\hline $\begin{array}{l}\text { IMP-like-1 } \\
\text { (IMP-81) }\end{array}$ & 246 & Subclass B1 metallo-beta-lactamase IMP-15 [Pseudomonas aeruginosa] & 96 & WP_063860575.1 & Beta-lactams \\
\hline qacE-like-1 & 110 & QacE [uncultured bacterium] & 81 & ACN22612.1 & Disinfectants \\
\hline qacG-like-1 & 96 & QacG, partial [uncultured bacterium] & 90 & ACS73614.1 & Disinfectants \\
\hline arr-like-1 & 150 & $\begin{array}{l}\text { NAD(+)--rifampin ADP-ribosyltransferase Arr-6 } \\
{[\text { Pseudomonas aeruginosa] }}\end{array}$ & 86 & WP_063842214.1 & Rifampicin \\
\hline sul $4^{\#}$ & 280 & Dihydropteroate synthase [Ardenticatena] & 68 & CUS02277.2 & Sulfonamides \\
\hline Dfr-like 1 & 107 & Dihydrofolate reductase [Pseudomonas aeruginosa] & 88 & BAT62904.1 & Trimethoprim \\
\hline Dfr-like 2 & 157 & Dihydrofolate reductase, partial [Vibrio cholerae] & 89 & AAT37842.1 & Trimethoprim \\
\hline
\end{tabular}

*The sequence is $100 \%$ identical to $b / a_{\mathrm{RSA} 1}$

"The sequence is $100 \%$ identical to sul4 


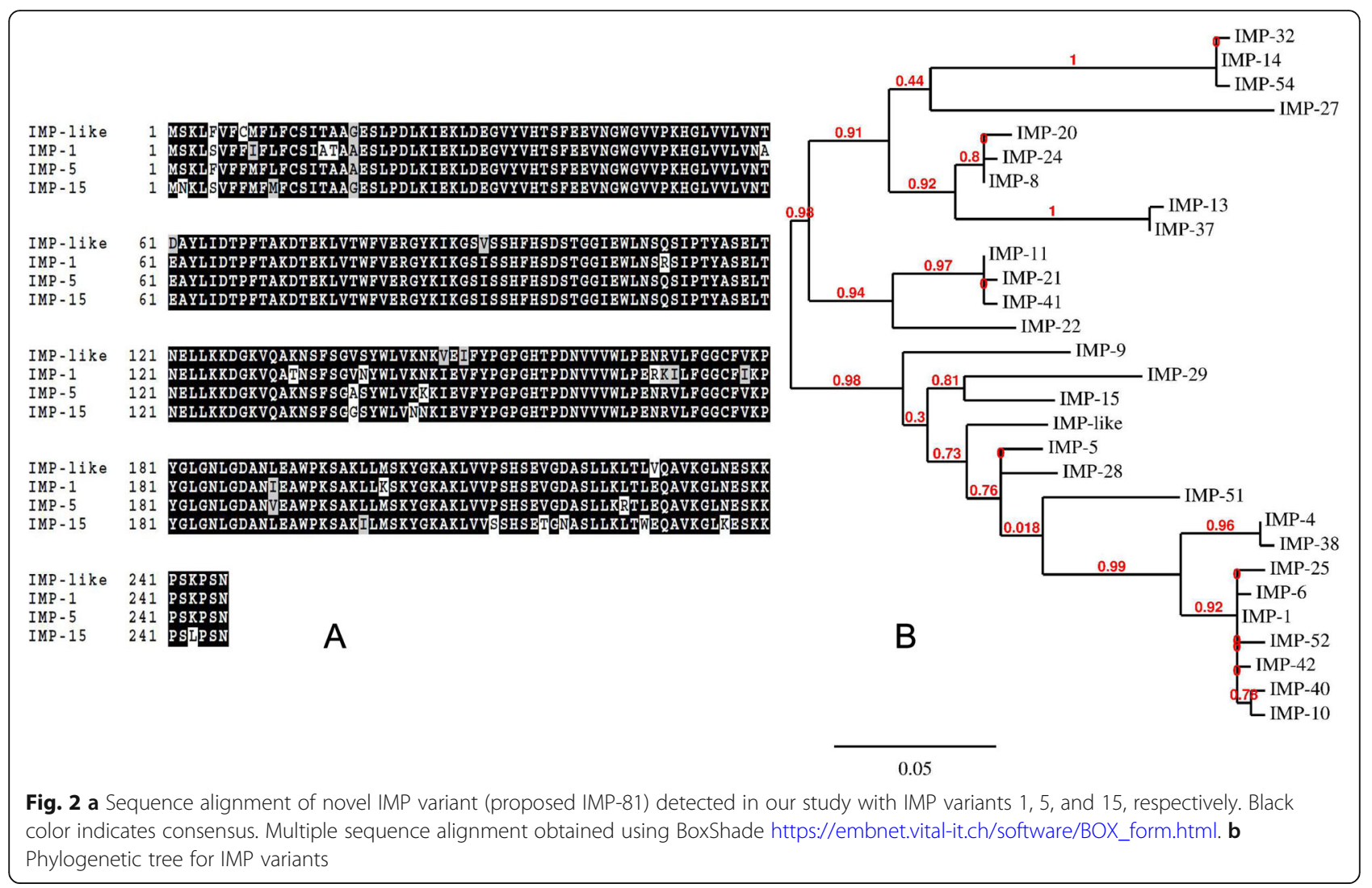

IMP-1, IMP-15, VIM-2, DIM-1, POM-1, and L1, respectively. We detected nine putative novel subclass $\mathrm{B} 1$, one novel subclass B2, and 10 novel subclass B3 carbapenemases, respectively. The list of these genes and the closest blast hits are presented in Table 2. The putative novel subclass B2 gene (2N30) was expressed in E. coli, and the CarbaNP test confirmed its ability to hydrolyze imipenem. Seven of the novel predicted B3 carbapenemases were synthesized and tested for imipenem degradation using CarbaNP test. Six out of the seven genes were positive for the test (Table 2). One of the subclass B1(1N7) protein falls in the same phylogenetic cluster as SPS-1 (Additional file 10: Figure S2). This enzyme has a substitution at position 116 (histidine at position 116 is replaced by a glycine), consistent with other enzymes belonging to this phylogenetic cluster [10].

\section{Discussion}

In order to identify novel resistant determinants, particularly carbapenemases, we explored sewage effluent collected from a hospital in India, a country with high use of carbapenems and widespread problems with carbapenemase resistance. Using both hidden Markov models on shotgun data, as well as amplicon sequencing of integron gene cassettes, we found several novel ARGs. These include several different carbapenemases that also turned out to be functional when expressed in E. coli.
The present study therefore expands our knowledge of novel resistance genes. The co-occurrence of pathogens and novel resistance genes to critically important antibiotics offers increased opportunities for unwanted horizontal gene transfer events. Hence, the studied hospital sewage environment appears to provide an example of the "ecological connectivity" [20] needed for genes to move across niches and environments to eventually become clinical problems.

We demonstrate the presence of a functional novel variant of a mobile IMP-type (proposed IMP-81) carbapenemase as a gene cassette within a class 1 integron. IMP-1 was the first mobile MBL detected and is one of the five major clinical carbapenemases found globally $[44,45]$. Identification of this novel variant could reflect that MBLs are constantly evolving. Indeed, its presence in sewage from an Indian hospital could potentially be linked to its evolution as a result of high consumption of broad-spectrum antibiotics, including carbapenems, in India $[1,46]$. Using shotgun metagenomics and computational models, we have substantially increased our understanding about the diversity of MBLs. Although several studies have detected known MBLs in hospital effluent, to the best of our knowledge, none of the studies have detected as many novel MBLs in hospital effluent to date [28, 30, 47]. We extended our previous subclass B1 model for the detection of subclass B3 
Table 2 The list of MBLs detected using computational model and shotgun sequence data

\begin{tabular}{|c|c|c|c|c|c|}
\hline Gene name & MBL subclass & Length aa & Closest homolog in NCBI protein database & $\%$ identity aa & Accession number \\
\hline DIM & B1 & 202 & Subclass B1 metallo-beta-lactamase DIM-1 [Pseudomonas stutzeri] & 100.0 & WP_063860203.1 \\
\hline NDM & B1 & 248 & Metallobetalactamase NDM-1 [Klebsiella pneumoniae] & 100.0 & AGC54622.1 \\
\hline IMP & B1 & 240 & Beta-lactamase IMP-1 precursor [Pseudomonas aeruginosa] & 100.0 & CRX26419.1 \\
\hline VIM & B1 & 248 & Subclass B1 metallo-beta-lactamase VIM-2 [Pseudomonadales] & 100.0 & WP_003108247.1 \\
\hline IMP & B1 & 240 & Subclass B1 metallo-beta-lactamase IMP-15 [Pseudomonas aeruginosa] & 100.0 & WP_063860575.1 \\
\hline $1 \mathrm{~N} 26$ & B1 & 241 & Beta-lactamase [uncultured bacterium] & 74.79 & ALG03680.1 \\
\hline $1 \mathrm{~N} 27$ & B1 & 242 & Subclass B1 metallo-beta-lactamase [bacterium 336/3] & 71.97 & WP_054042800.1 \\
\hline $2 \mathrm{~N} 30^{*}$ & B2 & 252 & ChpA family subclass B2 metallo-beta-lactamase [Aeromonas lacus] & 51.48 & WP_033113784.1 \\
\hline 1 N32 & B1 & 241 & $\begin{array}{l}\text { Hypothetical protein A3D31_07435 [Fluviicola sp. } \\
\text { RIFCSPHIGHO2_02_FULL_43_260] }\end{array}$ & 55.04 & OGS79779.1 \\
\hline $1 \mathrm{~N} 4$ & B1 & 247 & Subclass B1 metallo-beta-lactamase [bacterium 336/3] & 73.55 & WP_054042800.1 \\
\hline $1 \mathrm{~N} 59$ & B1 & 244 & Hypothetical protein A2041_05420 [Bacteroidetes bacterium GWA2_31_9b] & 52.7 & OFX20903.1 \\
\hline $1 \mathrm{~N} 6$ & B1 & 246 & Subclass B1 metallo-beta-lactamase [bacterium 336/3] & 59.5 & WP_054042800.1 \\
\hline $1 \mathrm{~N} 8$ & B1 & 234 & Subclass B1 metallo-beta-lactamase [Flectobacillus major] & 70.67 & WP_044171073.1 \\
\hline $1 \mathrm{~N} 9$ & B1 & 240 & Beta-lactamase [uncultured bacterium] & 66.53 & ALG03680.1 \\
\hline $1 \mathrm{~N} 7$ & B1 & 273 & Hypothetical protein Gferi_08260 [Geosporobacter ferrireducens] & 48.0 & AOT69571.1 \\
\hline POM-1 & B3 & 223 & B3 beta-lactamase [Pseudomonas otitidis] & 100.0 & ADC79563.1 \\
\hline$L-1$ & B3 & 210 & $\begin{array}{l}\text { LW82289.1 metallo-beta-lactamase L1 family protein } \\
\text { [Acinetobacter sp. WC-743] }\end{array}$ & 99.52 & WP_009585815.1 \\
\hline $3 \mathrm{~N} 14$ & B3 & 283 & Subclass B3 metallo-beta-lactamase [Phenylobacterium sp. Root700] & 69.58 & WP_056733210.1 \\
\hline $3 N 32^{*}$ & B3 & 281 & BJP_beta_lactamase [uncultured bacterium] & 61.59 & AIA10847.1 \\
\hline $3 N 33$ & B3 & 289 & BJP_beta_lactamase [uncultured bacterium] & 61.4 & AIA10847.1 \\
\hline $3 \mathrm{~N}^{2} 0^{*}$ & B3 & 290 & Subclass B3 metallo-beta-lactamase [Phenylobacterium sp. Root700] & 69.96 & WP_056733210.1 \\
\hline $3 \mathrm{~N} 51^{*}$ & B3 & 299 & Subclass B3 metallo-beta-lactamase [Novosphingobium sp. PP1Y]C & 70.57 & WP_013834039.1 \\
\hline $3 \mathrm{~N} 55^{*}$ & B3 & 284 & Subclass B3 metallo-beta-lactamase [Phenylobacterium sp. Root700]K & 70.82 & WP_056733210.1 \\
\hline $3 \mathrm{~N} 61^{*}$ & B3 & 297 & Subclass B3 metallo-beta-lactamase [Croceicoccus marinus] & 57.65 & WP_066847047.1 \\
\hline $3 \mathrm{~N} 73^{\#}$ & B3 & 295 & BJP_beta_lactamase [uncultured bacterium] & 62.98 & AIA10847.1 \\
\hline $3 N 8$ & B3 & 297 & Subclass B3 metallo-beta-lactamase [Sphingomonadaceae]E & 55.85 & WP_008831296.1 \\
\hline $3 N 1^{*}$ & B3 & 300 & Subclass B3 metallo-beta-lactamase [Novosphingobium sp. Leaf2] & 53.0 & WP_056771586.1 \\
\hline
\end{tabular}

*These genes were positive in the CarbaNP test

"This gene was negative in the CarbaNP test

MBLs and found 2 known and 10 putative novel B3 carbapenemases. Six out of seven predicted and synthesized B3 genes were functional in E. coli, thus demonstrating the high accuracy of our approach. Four of the predicted B3 genes belong to the same part of the phylogenetic tree as the clinically important L1 betalactamase and the only known version of B3 carbapenemases located on a plasmid, AIM-1 [48, 49]. Although these genes are functional in E. coli, the computational prediction does not provide information about their genetic context. Hence, we do not know their potential for mobility nor the host species for these genes. Further investigation of genetic context and host species identification is thus warranted.

NDM was the most abundant of the clinical carbapenemase detected in the effluent. This finding is in accordance with the clinical data on carbapenemase producing pathogens from Mumbai [50]. A recent study showed that NDM-1 is also common in hospital effluents from Delhi, India [30]. Interestingly, we detected OXA-58 as the second most abundant carbapenemase gene. OXA-58 has been found on a plasmid in Enterobacteriaceae, and it is a mobile carbapenemase regularly encountered in Acinetobacter [51, 52]. A previous study of ours showed that the abundance of OXA-58 in Indian river sediments contaminated with untreated urban waste strongly correlates with the abundance of Acinetobacter [53]. Similarly, the high abundance of OXA-58 in the hospital effluent detected here can be explained by a high abundance of Acinetobacter. The rates of $A$. baumannii nosocomial infections have risen globally in past decades [54]. Acinetobacter is invariably resistant to 
multiple antibiotics, including last resort drugs like carbapenems and colistin, making treatment difficult. Acinetobacter often carries conjugative plasmids bearing multidrug resistance markers and carbapenemases belonging to the OXA-type as well as MBLs. Acinetobacter can readily exchange these plasmids carrying drug resistance markers with members of family Enterobacteriaceae [51, 55].

GES-type beta-lactamases were the second most abundant ESBLs in our study, with several variants capable of low-level hydrolysis of carbapenems [56]. GES-carrying carbapenem-resistant strains have been previously isolated from hospital effluent [57]. GES-type ESBL genes are found globally and exclusively as integron gene cassettes in Gram-negative pathogens, including $P$. aeruginosa, E. coli, K. pneumoniae, and A. baumannii [58]. The high abundance of GES-type beta-lactamases in the shotgun dataset can at least partly be explained by high abundance of class 1 integrons in our samples. The recently discovered ESBL gene $b l a_{\mathrm{RSA} 1}$ was also detected in integron gene cassettes here. The $b l a_{\mathrm{RSA} 1}$ gene is phylogenetically close to GES-type ESBLs. The betalactam hydrolytic profile of the $b l a_{\mathrm{RSA} 1}$ protein resembled that of GES-2. Although $b l a_{\mathrm{RSA} 1}$ does not hydrolyze carbapenems, there is a possibility that natural mutants might occur which may possess this activity, as is the case for other GES variants [13]. The same may apply for other ESBLs, as was recently demonstrated by the discovery of a natural mutant of OXA-10 with increased carbapenemase activity in Swedish hospital effluent [59].

Sulfonamide resistance genes are common in domestic and hospital effluents [60]. Our results showed that sul1 was the most abundant mobile resistance gene, which is in accordance with a previous study of hospital wastewater [60]. The recently described novel sulfonamide resistance gene sul4 was detected along with the ISCR20 transposes as described earlier [21]. Both sul4 and $b l a_{\mathrm{RSA} 1}$ were first described from river sediments contaminated with waste from drug manufacturing plants near Hyderabad, India, and concerns were raised about finding these genes in clinical isolates $[13,21]$. The presence of these genes in integron gene cassettes from hospital effluent suggests that these genes are accessible to pathogens and might already have made their way into human pathogens. This finding also emphasizes the need to explore and characterize environmental ARGs, which may end up in clinics in the future.

\section{Conclusions}

We show that explorative studies for novel antibiotic resistance determinant in hospital effluent can contribute to early identification of what may become future clinical problems. An expanded knowledge of these novel genes can facilitate actions to mitigate their potential spread in the clinic. Bacteria carrying novel ARGs, including novel carbapenemases, co-exist with pathogens, thus creating a niche where the acquisition of novel ARGs by pathogens may take place. It is also possible that such gene exchange is further boosted by the expected presence of antibiotic residues [61, 62]. If released untreated or inadequately treated, as in many low- and middle-income countries, there is increased opportunities for transmission of enteric pathogens, including resistant ones [30]. Hence, hospital effluent discharges also deserve attention from antimicrobial resistance risk management point of view.

\section{Methods}

\section{Sampling, DNA extraction, and shotgun sequencing}

Effluent samples were collected directly from the sewer line from a hospital in Mumbai, India, on 2 different days (30 October 2014 and 1 November 2014). Each sample comprised composite sample collected every hour (100 $\mathrm{ml}$ each time) during the day from 8 a.m. to 6 p.m. in a sterile plastic bottle. The sub-samples were stored at a temperature of $4{ }^{\circ} \mathrm{C}$, mixed together and filtered on the same day using a $0.2-\mu \mathrm{M}$ filter. DNA was extracted from the filters using QIAamp DNA Stool Mini Kit (Qiagen, Germany). The DNA was quantified using dsDNA High Sensitivity (HS) Assay kit on the Qubit $^{\circ}$ Fluorometer (Invitrogen, USA) and stored at $20^{\circ} \mathrm{C}$. The DNA was sent for shotgun metagenomic sequencing (paired-end, 125 base pair reads) on Illumina HiSeq2500 platform at Science for Life Laboratories (Stockholm, Sweden).

\section{Sequence analysis of shotgun metagenomic data}

The sequence analysis was performed according to the protocol described by Marathe et al. [53]. In brief, the sequences were trimmed for adaptors and quality-filtered using Trim Galore (http://www.bioinformatics.babraham. ac.uk/projects/trim_galore/) with a phred quality score of 28 and a maximum error rate (the number of errors divided by the length of the matching region) of 0.1. The quality-processed reads from the metagenomes were mapped against protein sequences from a high-quality and manually curated database of mobile ARGs and mobile genetic elements, Resqu database (Resqu database; version 1.1; 1928 Diagnostics, Gothenburg; http://www. 1928diagnostics.com/resdb), which contains ARGs that have been previously reported to be horizontally transferred or carried on a mobile genetic element. Full-length coverage of the query reads was set against target resistance genes with a sequence identity threshold of $90 \%$, and only the best hits were retrieved (options "-usearch_global -id 0.9 maxaccepts 1 -threads 16"). The list of resistance genes in the Resqu database is given in Additional file 7 : Table S7. Analysis of bacterial virulence-associated genes (virulence factors) in the metagenomes was performed 
using a set of experimentally verified virulence factors collected from the Virulence Factor Database (http:// www.mgc.ac.cn/VFs/) [63]. To characterize the overall taxonomic distribution, quality-filtered shotgun reads were used as input to extract the reads corresponding to small subunit (SSU) 16S bacterial ribosomal RNA genes from the metagenomes and assigned them to different taxonomic groups using Metaxa2 (version 2.1) with default options [64].

\section{PCR amplification and sequencing of integron gene cassettes}

Amplification of integron gene cassettes was performed according to the protocol described by Razavi et al. [21]. In brief, integron gene cassettes were amplified from the hospital effluent DNA using three sets of primers previously described using phusion taq polymerase (thermoscientific, USA). PCR products were purified using QIAquick PCR Purification Kit (Qiagen, Germany) and quantified using the Qubit ${ }^{\odot}$ Fluorometer (Invitrogen, USA). The purified PCR products were sent for singlemolecule real-time (SMRT) sequencing technology (Pacific Biosciences) and shotgun metagenomic sequencing to produce (paired-end 250 base pair reads on the Illumina MiSeq platform) at Science for Life Laboratories in Uppsala and Stockholm, respectively.

\section{Sequence analysis of integron gene cassettes}

Sequence analysis was performed using the method described by Razavi et al., 2017. In brief, quality-filtered PacBio reads were corrected using Illumina reads with a hybrid correction pipeline for SMRT sequencing, i.e., Proovread [65]. Reads were clustered at $100 \%$ identity using CD-HIT to remove redundancy. The open reading frames (ORFs) were predicted using Prodigal [66] and annotated through similarity searches against the NCBI non-redundant protein (nr) databases (13 April 2017). Putative novel resistance genes were identified based on their sequence identity and the length of the alignment (coverage) to known homologs genes both in the CARD (version 1.1.0, REF) and the NCBI nr protein databases. Integron gene cassettes are expected to carry a wide array of genes, including ARGs. Hence CARD and NCBI (nr), which are broader databases compared to Resqu, were used for characterizing integron gene cassettes. We classified ORFs with at least 95\% identity to closest homologs in the CARD database as "known resistance genes." Although the exact cutoff is subjective, this has been used by others in the past [67]. We classified ORFs with a best match to a resistance gene in the CARD database as "putative novel resistance genes" if they had an identity below $95 \%$ and a coverage greater than $65 \%$ [21]. The gene cassettes with known functions were clustered at $99 \%$ identity cutoff to remove redundancy using CD-HIT. HattCI was used to identify the attachment site $a t t C$ in the reads [68].

\section{Prediction of class B beta-lactamases}

The novel MBL genes were identified from shotgun sequence data using a computational method based on a hidden Markov model (HMM) reported recently [5, 10]. For identification of subclasses B1 and B2, the model was built using HMMER (version 3.1b1) and trained using 20 verified genes in the B1 MBLs subclass, while the model for subclass B3 was trained using 11 verified genes belonging to subclass B3. The list of the genes is represented in Additional file 8: Table S8. The subclasses $\mathrm{B} 1$ and B2 are rather similar while B3 is distinctly different, based on sequence identity. Also, phylogenetic evidence suggests that the resistance mechanisms of the subclasses B1, B2, and B3 may have developed independently $[69,70]$. Hence, one model can detect both B1 and B2 genes, while a different model was created for B3 genes. The created and optimized models were applied directly to the fragmented data (short reads) and the fragments predicted to belong to either subclass B1, B2, or B3 were retrieved and assembled into full-length genes using SPAdes version 3.8.1 with parameter "-meta" [71]. The full-length genes were then once again subjected to the hidden Markov models, this time using a threshold score optimized for full-length genes. The genes that passed the final classification step were retrieved and clustered at a $70 \%$ amino acid sequence similarity together with previously characterized MBLs using USEARCH with parameters "-cluster_fast -id 0.7" [72]. The list of previously characterized MBLs used in clustering can be seen in Additional file 8: Table S8.

\section{Functional verification of candidate novel resistance genes}

For each antibiotic class (except aminoglycosides, as the expression vector contained a kanamycin resistance gene), we selected one putative novel resistance gene/ gene variant detected in integron gene cassettes for functional verification. Among the genes that had their best match to a known resistance gene, we chose the one with the lowest identity to a known ARG for each antibiotic class. Genes with $>95 \%$ identity to a resistance gene in CARD were not tested, with the exception of a putative novel IMP gene that had $96 \%$ identity to a known ARG. The reason for this is that carbapenemase genes are clinically very important and even small changes in MBL protein sequence may change their hydrolytic profile [73]. With regards to novel MBLs derived from the HMM analyses, seven novel genes from different clades of phylogenetic tree representing subclass B3 were selected for functional verification along 
with one gene representing subclass B2. The candidate novel genes were synthesized at ThermoFisher Scientific, Germany, using their GeneArt Gene Synthesis service and subcloned into the expression vector pZE21-MCS1 as described previously [13]. The plasmids containing novel resistance gene candidates were then transformed into E. coli C600Z1 (Expressys, Germany) by electroporation. The minimum inhibitory concentrations (MICs) of the respective antibiotics for the E. coli strains containing synthesized candidate novel resistance genes were determined using E-tests on Mueller-Hinton Agar plates (BioMérieux, France) with the addition of $100 \mathrm{ng} / \mu \mathrm{l}$ anhydrotetracycline as an inducer of the expression. E. coli strain containing an empty vector was used as a negative control. For verification of a putative novel IMP variant and MBLs, CarbaNP test was carried out as described earlier [74]. CarbaNP test is a biochemical test based on the detection of the acidification resulting from imipenem hydrolysis by carbapenemases [74].

\section{De novo assembly of Acinetobacter baumannii reads}

Illumina reads that aligned with at least $90 \%$ identity to the nucleotide sequence of the type OXA-51 gene from $A$. baumannii strain AB030 (accession NZ_CP009257.1) were identified using BBMap (v38.32, "maxindel=2 minid $=0.90$ idfilter $=0.90$ strictmaxindel") and were then assembled using MEGAHIT (v1.1.3, defaults) [75, 76]. The resulting 1341 base pair contig with a 26-fold coverage was searched against NCBI database using Entrez (18 Jan. 2019), and the genomes which contained highly similar homologs were selected (accessions NZ_KB849297.1, NZ_KB849308.1, NZ_CP033754.1, NZ_CP022283.1, NZ_ CP027530.1, NZ_CP018332.1, NZ_CP020597.1, NZ_ LN997846.1, NZ_LN865143.1). These were again used to recruit read pairs using BBMap (previous parameters) and then assembled using MEGAHIT (previous parameters).

\section{Additional files}

Additional file 1: Table S1. Relative abundance (resistance gene copies per $16 \mathrm{~S}$ rRNA gene) of antibiotic resistance genes and mobile genetic elements detected in our study. (XLSX 13 kb)

Additional file 2: Table S2. List of virulence genes detected in our study and their relative abundance to 165 rRNA genes. (XLSX $18 \mathrm{~kb}$ )

Additional file 3: Table S3. List of bacterial genera detected in our study and their relative abundance as a percentage of all 16S rRNA gene reads. (XLSX $48 \mathrm{~kb}$ )

Additional file 4: Table S4. List of genes detected in class 1 integron gene cassettes. (XLSX 2620 kb)

Additional file 5: Table S5. List of known and putative novel ARGs detected in class 1 integron gene cassettes. (XLSX 57 kb)

Additional file 6: Table S6. The MIC values in micrograms per milliliter for $E$. coli strains containing synthesized putative novel antibiotic resistance genes, against respective antibiotics. (DOCX $13 \mathrm{~kb}$ )

Additional file 7: Table S7. List of resistance genes in the Resqu database. (XLSX $24 \mathrm{~kb}$ )
Additional file 8: Table S8. List of resistance genes used for building model for prediction of class B MBLs and list of previously characterized genes used for clustering. (XLSX $13 \mathrm{~kb}$ )

Additional file 9: Figure S1. Alignment of assembled contigs with $A$. baumannii strain 2600 genome. (TIF 404 kb)

Additional file 10: Figure S2. A phylogenetic tree describing the evolutionary relationship between the subclass B1/B2 MBLs detected in this study. (PDF $117 \mathrm{~kb}$ )

Additional file 11: Figure S3. A phylogenetic tree describing the evolutionary relationship between the subclass B3 MBLs detected in this study. (PDF $43 \mathrm{~kb}$ )

\section{Acknowledgements}

Sequencing was conducted at the Swedish National Genomics Infrastructure (NGI) at the Science for Life Laboratories in Stockholm. Computational analysis was performed on resources provided by the Swedish National Infrastructure for Computing (SNIC) at Chalmers Centre for Computational Science and Engineering (C3SE) cluster.

\section{Authors contributions}

NPM and DGJL conceived and designed the study. SS collected the samples. NPM performed DNA extractions, PCR analysis, and functional verification of the novel genes and drafted the manuscript. MR performed bioinformatic analysis of gene cassette data, and FB performed bioinformatic analysis and gene prediction using computational models, while CP analyzed shotgun data and JD was responsible for assemblies. EK assisted with bioinformatic analyses and edited the manuscript; NP drafted the manuscript with significant input from DGJL. All authors read and approved the final manuscript.

\section{Funding}

This work was funded by the Swedish Research Council for Environment, Agriculture and Spatial Planning (FORMAS) (2012-86 to D.G.J.L), the Swedish Research Council (VR) (2015 02492 and 2018-05771 to D.G.J.L), and the Centre for Antibiotic Resistance Research (CARe) at the University of Gothenburg.

\section{Availability of data and materials}

The raw sequencing data of hospital effluent have been deposited in the NCBI Sequence Read Archive (SRA) under the bio-project PRJNA497765. The gene sequences for novel MBLs have been submitted to GenBank under accession numbers MN017279-MN017299.

Ethics approval and consent to participate

Not applicable

\section{Consent for publication}

Not applicable

\section{Competing interests}

The authors declare no competing financial interests.

\section{Author details}

${ }^{1}$ Centre for Antibiotic Resistance Research (CARe), University of Gothenburg, Gothenburg, Sweden. ${ }^{2}$ Department of Infectious Diseases, Institute of Biomedicine, Sahlgrenska Academy, University of Gothenburg, Gothenburg, Sweden. ${ }^{3}$ Institute of Marine Research (IMR), Bergen, Norway. ${ }^{4}$ Department of Mathematical Sciences, Chalmers University of Technology and University of Gothenburg, Gothenburg, Sweden. ${ }^{5}$ Plant Health and Environment Laboratory (PHEL), Ministry for Primary Industries (MPI), Auckland, New Zealand. ${ }^{6}$ Mahatma Gandhi Mission medical college, Navi Mumbai, Maharashtra, India.

Received: 11 February 2019 Accepted: 5 June 2019 Published online: 27 June 2019

\section{References}

1. Laxminarayan R, Chaudhury RR. Antibiotic resistance in India: drivers and opportunities for action. PLoS Med. 2016;13(3):e1001974. 
2. Wellington EMH, Boxall ABA, Cross P, Feil EJ, Gaze WH, Hawkey PM, Johnson-Rollings AS, Jones DL, Lee NM, Otten W, et al. The role of the natural environment in the emergence of antibiotic resistance in gramnegative bacteria. Lancet Infect Dis. 2013;13(2):155-65.

3. Bengtsson-Palme J, Kristiansson E, Larsson DGJ. Environmental factors influencing the development and spread of antibiotic resistance. FEMS Microbiol Rev. 2017. https://doi.org/10.1093/femsre/fux053.

4. Gibson MK, Forsberg KJ, Dantas G. Improved annotation of antibiotic resistance determinants reveals microbial resistomes cluster by ecology. ISME J. 2015;9(1):207.

5. Berglund F, Österlund T, Boulund F, Marathe NP, Larsson DJ, Kristiansson E. Identification and reconstruction of novel antibiotic resistance genes from metagenomes. Microbiome. 2019;7(1):52.

6. Yang Y, Jiang X, Chai B, Ma L, Li B, Zhang A, Cole JR, Tiedje JM, Zhang T. ARGs-OAP: online analysis pipeline for antibiotic resistance genes detection from metagenomic data using an integrated structured ARG-database. Bioinformatics. 2016;32(15):2346-51

7. McArthur AG, Waglechner N, Nizam F, Yan A, Azad MA, Baylay AJ, Bhullar K, Canova MJ, De Pascale G, Ejim L. The comprehensive antibiotic resistance database. Antimicrob Agents Chemother. 2013;57(7):3348-57.

8. Boulund F, Berglund F, Flach C-F, Bengtsson-Palme J, Marathe NP, Larsson $D G J$, Kristiansson E. Computational discovery and functional validation of novel fluoroquinolone resistance genes in public metagenomic data sets. BMC Genomics. 2017;18(1):682.

9. Boulund F, Johnning A, Pereira MB, Larsson DGJ, Kristiansson E. A novel method to discover fluoroquinolone antibiotic resistance (qnr) genes in fragmented nucleotide sequences. BMC Genomics. 2012;13.

10. Berglund F, Marathe NP, Österlund T, Bengtsson-Palme J, Kotsakis S, Flach CF, Larsson DGJ, Kristiansson E. Identification of 76 novel B1 metallo- $\beta$ lactamases through large-scale screening of genomic and metagenomic data. Microbiome. 2017;5(1):134

11. Horan $\mathrm{K}$, Shelton CR, Girke T. Predicting conserved protein motifs with SubHMMs. BMC Bioinformatics. 2010;11(1):205.

12. Mullany P. Functional metagenomics for the investigation of antibiotic resistance. Virulence. 2014;5(3):443-7.

13. Marathe NP, Janzon A, Kotsakis SD, Flach C-F, Razavi M, Berglund F, Kristiansson $E$, Larsson DGJ. Functional metagenomics reveals a novel carbapenem-hydrolyzing mobile beta-lactamase from Indian river sediments contaminated with antibiotic production waste. Environ Int. 2018;112:279-86.

14. Flach C-F, Boulund F, Kristiansson E, Larsson DGJ. Functional verification of computationally predicted qnr genes. Ann Clin Microbiol Antimicrob. 2013; 12:34.

15. Sommer MO, Dantas G, Church GM. Functional characterization of the antibiotic resistance reservoir in the human microflora. Science. 2009; 325(5944):1128-31.

16. Amos GC, Zhang L, Hawkey PM, Gaze WH, Wellington EM. Functional metagenomic analysis reveals rivers are a reservoir for diverse antibiotic resistance genes. Vet Microbiol. 2014;171(3-4):441-7.

17. Forsberg KJ, Patel S, Gibson MK, Lauber CL, Knight R, Fierer N, Dantas G. Bacterial phylogeny structures soil resistomes across habitats. Nature. 2014; 509(7502):612-6

18. Hatosy SM, Martiny AC, Nojiri $H$. The ocean as a global reservoir of antibiotic resistance genes. Appl Environ Microbiol. 2015;81(21):7593-9.

19. Bengtsson-Palme J, Larsson DGJ. Antibiotic resistance genes in the environment: prioritizing risks. Nat Rev Microbiol. 2015;13(6):396.

20. Martínez JL, Coque TM, Baquero F. What is a resistance gene? Ranking risk in resistomes. Nat Rev Microbiol. 2015;13(2):116-23.

21. Razavi M, Marathe NP, Gillings MR, Flach C-F, Kristiansson E, Larsson DGJ. Discovery of the fourth mobile sulfonamide resistance gene. Microbiome. 2017:5:160.

22. Mazel D. Integrons: agents of bacterial evolution. Nat Rev Microbiol. 2006; 4(8):608-20.

23. Gillings M, Boucher $\mathrm{Y}$, Labbate M, Holmes A, Krishnan S, Holley M, Stokes HW. The evolution of class 1 integrons and the rise of antibiotic resistance. J Bacteriol. 2008;190(14):5095-100.

24. Gillings MR. Integrons: past, present, and future. Microbiol Mol Biol Rev. 2014;78(2):257-77

25. Bengtsson-Palme J, Larsson DJ. Concentrations of antibiotics predicted to select for resistant bacteria: proposed limits for environmental regulation. Environ Int. 2016;86:140-9.
26. Rodriguez-Mozaz S, Chamorro S, Marti E, Huerta B, Gros M, Sànchez-Melsió A, Borrego CM, Barceló D, Balcázar JL. Occurrence of antibiotics and antibiotic resistance genes in hospital and urban wastewaters and their impact on the receiving river. Water Res. 2015;69:234-42.

27. Lien LTQ, Hoa NQ, Chuc NTK, Thoa NTM, Phuc HD, Diwan V, Dat NT, Tamhankar AJ, Lundborg CS. Antibiotics in wastewater of a rural and an urban hospital before and after wastewater treatment, and the relationship with antibiotic use-a one year study from Vietnam. Int J Environ Res Public Health. 2016;13(6):588

28. Hocquet $D$, Muller A, Bertrand X. What happens in hospitals does not stay in hospitals: antibiotic-resistant bacteria in hospital wastewater systems. J Hospital Infect. 2016;93(4):395-402.

29. Weingarten RA, Johnson RC, Conlan S, Ramsburg AM, Dekker JP, Lau AF, Khil P, Odom RT, Deming C, Park M. Genomic analysis of hospital plumbing reveals diverse reservoir of bacterial plasmids conferring carbapenem resistance. MBio. 2018;9(1):e02011-7.

30. Lamba M, Graham DW, Ahammad SZ. Hospital wastewater releases of carbapenem-resistance pathogens and genes in urban India. Environ Sci Technol. 2017:51(23):13906-12

31. Rowe WP, Baker-Austin C, Verner-Jeffreys DW, Ryan JJ, Micallef C, Maskell DJ, Pearce GP. Overexpression of antibiotic resistance genes in hospital effluents over time. J Antimicrob Chemother. 2017;72(6):1617-23.

32. Buelow E, Bayjanov JR, Willems RJ, Bonten MJ, Schmitt H, Van Schaik W. The microbiome and resistome of hospital sewage during passage through the community sewer system. bioRxiv. 2017:216242.

33. Kakkar M, Walia K, Vong S, Chatterjee P, Sharma A. Antibiotic resistance and its containment in India. BMJ. 2017;358.

34. Hellen Gelband MM-P, Pant S, Gandra S, Levinson J, Barter D, White A, Laxminarayan R. The state of the world's antibiotics, 2015. Washington DC: Center for Disease Dynamics, Economics \& Policy; 2015.

35. Vetrovský $T$, Baldrian $P$. The variability of the $165 \mathrm{rRNA}$ gene in bacterial genomes and its consequences for bacterial community analyses. PloS One. 2013:8(2):e57923.

36. Klappenbach JA, Saxman PR, Cole JR, Schmidt TM. rrndb: the ribosomal RNA operon copy number database. Nucleic Acids Res. 2001;29(1):181-4.

37. Mellies JL, Barron AM, Carmona AM. Enteropathogenic and enterohemorrhagic Escherichia coli virulence gene regulation. Infect Immun. 2007;75(9):4199-210.

38. Zhang H, Zhou Y, Bao H, Liu H-w. Vi antigen biosynthesis in Salmonella typhi: characterization of UDP-N-acetylglucosamine C-6 dehydrogenase (TviB) and UDP-N-acetylglucosaminuronic acid C-4 epimerase (TviC). Biochemistry. 2006;45(26):8163-73.

39. Palmqvist N, Josefsson E, Tarkowski A. Clumping factor A-mediated virulence during Staphylococcus aureus infection is retained despite fibrinogen depletion. Microbes and infection. 2004;6(2):196-201.

40. Courtney HS, Dale JB, Hasty D. Differential effects of the streptococcal fibronectin-binding protein, FBP54, on adhesion of group A streptococci to human buccal cells and HEp-2 tissue culture cells. Infection and immunity. 1996;64(7):2415-9

41. Turton JF, Woodford N, Glover J, Yarde S, Kaufmann ME, Pitt TL. Identification of Acinetobacter baumannii by detection of the blaOXA-51like carbapenemase gene intrinsic to this species. Journal of clinical microbiology. 2006;44(8):2974-6.

42. Stoddard SF, Smith BJ, Hein R, Roller BR, Schmidt TM. rrn DB: improved tools for interpreting rRNA gene abundance in bacteria and archaea and a new foundation for future development. Nucleic acids research. 2014;43(D1):D593-8.

43. Cabanettes F, Klopp C. D-Genies: dot plot large genomes in an interactive, efficient and simple way. PeerJ [Internet. 2018;6:e4958.

44. Watanabe M, lyobe $\mathrm{S}$, Inoue M, Mitsuhashi S. Transferable imipenem resistance in Pseudomonas aeruginosa. Antimicrobial agents and chemotherapy. 1991;35(1):147-51.

45. van Duin D, Doi Y. The global epidemiology of carbapenemase-producing Enterobacteriaceae. Virulence. 2017:8(4):460-9.

46. Gandra S, Klein EY, Pant S, Malhotra-Kumar S, Laxminarayan R. Faropenem consumption is increasing in India. Clinical Infectious Diseases. 2016;62(8):1050-2.

47. White L, Hopkins K, Meunier D, Perry CL, Pike R, Wilkinson P, Pickup RW, Cheesbrough J, Woodford N. Carbapenemase-producing Enterobacteriaceae in hospital wastewater: a reservoir that may be unrelated to clinical isolates. J Hosp Infect. 2016:93(2):145-51.

48. Okazaki A, Avison MB. Induction of $L 1$ and $L 2 \beta$-lactamase production in Stenotrophomonas maltophilia is dependent on an AmpR-type regulator. Antimicrobial agents and chemotherapy. 2008;52(4):1525-8. 
49. Yong D, Toleman MA, Bell J, Ritchie B, Pratt R, Ryley H, Walsh TR. Genetic and biochemical characterization an acquired subgroup B3 metallo- $\beta$-lactamase gene, blaAIM-1, and its unique genetic context in Pseudomonas aeruginosa from Australia. Antimicrob Agents Chemother. 2012:AAC. 05654-11.

50. Kazi M, Drego L, Nikam C, Ajbani K, Soman R, Shetty A, Rodrigues C. Molecular characterization of carbapenem-resistant Enterobacteriaceae at a tertiary care laboratory in Mumbai. Eur J Clin Microbiol Infect Dis. 2015;34(3):467-72.

51. Khajuria A, Praharaj AK, Kumar M, Grover N. Molecular characterization of carbapenem resistant isolates of Acinetobacter baumannii in an intensive care unit of a tertiary care centre at central India. J Clin Diagn Res. 2014;8(5):DC38-40.

52. Coelho J, Woodford N, Afzal-Shah M, Livermore D. Occurrence of OXA-58like carbapenemases in Acinetobacter spp. collected over 10 years in three continents. Antimicrob Agents Chemother. 2006;50(2):756-8.

53. Marathe NP, Pal C, Gaikwad SS, Jonsson V, Kristiansson E, Larsson DGJ. Untreated urban waste contaminates Indian river sediments with resistance genes to last resort antibiotics. Water Res. 2017;124:388-97.

54. Antunes L, Visca P, Towner KJ. Acinetobacter baumannii: evolution of a global pathogen. Pathog Dis. 2014;71(3):292-301.

55. Fu $Y$, Du $X, J i$ J, Chen $Y$, Jiang $Y, Y u$ Y. Epidemiological characteristics and genetic structure of blaNDM-1 in non-baumannii Acinetobacter spp. in China. J Antimicrob Chemother. 2012:dks192.

56. Kotsakis SD, Miriagou V, Tzelepi E, Tzouvelekis LS. Comparative biochemical and computational study of the role of naturally occurring mutations at Ambler positions 104 and 170 in GES $\beta$-lactamases. Antimicrob Agents Chemother. 2010;54(11):4864-71.

57. Gomi R, Matsuda T, Yamamoto M, Chou P-H, Tanaka M, Ichiyama S, Yoneda M, Matsumura Y. Characteristics of carbapenemase-producing Enterobacteriaceae in wastewater revealed by genomic analysis. Antimicrob Agents Chemother. 2018:AAC. 02501-17.

58. Naas T, Poirel L, Nordmann P. Minor extended-spectrum $\beta$-lactamases. Clin Microbiol Infect. 2008;14(s1):42-52.

59. Kotsakis SD, Flach C-F, Razavi M, Larsson DJ. Characterization of the first OXA-10 natural variant with increased carbapenemase activity. Antimicrob Agents Chemother. 2019;63(1):e01817-8.

60. Le T-H, Ng C, Chen H, Yi XZ, Koh TH, Barkham TMS, Zhou Z, Gin KY-H. Occurrences and characterization of antibiotic resistant bacteria and genetic determinants of hospital wastewaters in a tropical country. Antimicrob Agents Chemother. 2016:AAC. 01556-16.

61. Jutkina J, Marathe N, Flach C-F, Larsson D. Antibiotics and common antibacterial biocides stimulate horizontal transfer of resistance at low concentrations. Sci Total Environ. 2018;616:172-8.

62. Diwan $\mathrm{V}$, Lundborg CS, Tamhankar AJ. Seasonal and temporal variation in release of antibiotics in hospital wastewater: estimation using continuous and grab sampling. PLoS One. 2013;8(7):e68715.

63. Chen L, Zheng D, Liu B, Yang J, Jin Q. VFDB 2016: hierarchical and refined dataset for big data analysis-10 years on. Nucleic Acids Res. 2016;44(D1): D694-7.

64. Bengtsson-Palme J, Hartmann M, Eriksson KM, Pal C, Thorell K, Larsson DGJ, Nilsson RH. METAXA2: improved identification and taxonomic classification of small and large subunit rRNA in metagenomic data. Mol Ecol Resour. 2015;15(6):1403-14.

65. Hackl T, Hedrich R, Schultz J, Förster F. proovread: large-scale high-accuracy PacBio correction through iterative short read consensus. Bioinformatics. 2014;30(21):3004-11.

66. Hyatt D, Chen G-L, LoCascio PF, Land ML, Larimer FW, Hauser LJ. Prodigal: prokaryotic gene recognition and translation initiation site identification. BMC Bioinformatics. 2010;11(1):119.

67. Munck C, Albertsen M, Telke A, Ellabaan M, Nielsen PH, Sommer MO. Limited dissemination of the wastewater treatment plant core resistome. Nat Commun. 2015;6.

68. Pereira MB, Wallroth M, Kristiansson E, Axelson-Fisk M. HattCl: fast and accurate attC site identification using hidden Markov models. J Comput Biol. 2016;23(11):891-902

69. Hall BG, Salipante SJ, Barlow M. The metallo- $\beta$-lactamases fall into two distinct phylogenetic groups. J Mol Evol. 2003;57(3):249-54.

70. Alderson RG, Barker D, Mitchell JB. One origin for metallo-ß-lactamase activity, or two? An investigation assessing a diverse set of reconstructed ancestral sequences based on a sample of phylogenetic trees. J Mol Evol. 2014;79(3-4):117-29.

71. Bankevich A, Nurk S, Antipov D, Gurevich AA, Dvorkin M, Kulikov AS, Lesin VM, Nikolenko SI, Pham S, Prjibelski AD. SPAdes: a new genome assembly algorithm and its applications to single-cell sequencing. J Comput Biol. 2012;19(5):455-77.

72. Edgar RC. Search and clustering orders of magnitude faster than BLAST. Bioinformatics. 2010:26(19):2460-1.

73. F Mojica M, A Bonomo R, Fast W: B1-Metallo- $\beta$-lactamases: where do we stand? Curr Drug Targets 2016, 17(9):1029-1050.

74. Dortet L, Poirel L, Errera C, Nordmann P. CarbAcineto NP test for rapid detection of carbapenemase-producing Acinetobacter spp. J Clin Microbiol. 2014;52(7):2359-64.

75. Bushnell B: BBMap short read aligner. University of California, Berkeley, California URL http://sourceforge.net/projects/bbmap 2016.

76. Li D, Liu C-M, Luo R, Sadakane K, Lam T-W. MEGAHIT: an ultra-fast singlenode solution for large and complex metagenomics assembly via succinct de Bruijn graph. Bioinformatics. 2015;31(10):1674-6.

\section{Publisher's Note}

Springer Nature remains neutral with regard to jurisdictional claims in published maps and institutional affiliations.
Ready to submit your research? Choose BMC and benefit from:

- fast, convenient online submission

- thorough peer review by experienced researchers in your field

- rapid publication on acceptance

- support for research data, including large and complex data types

- gold Open Access which fosters wider collaboration and increased citations

- maximum visibility for your research: over $100 \mathrm{M}$ website views per year

At $\mathrm{BMC}$, research is always in progress.

Learn more biomedcentral.com/submissions 